\title{
Large Scale Three-Dimensional Boundary Element Simulation of Subduction
}

\author{
Gabriele Morra ${ }^{1}$, Philippe Chatelain ${ }^{2}$, \\ Paul Tackley ${ }^{1}$, and Petros Koumoutsakos ${ }^{2}$ \\ ${ }^{1}$ Geophysical Fluid Dynamics, ETH Zurich \\ Einsteinstr. 1, Zurich CH-80XX, Switzerland \\ http://www.gfd.ethz.ch \\ ${ }^{2}$ Computational Science and Engineering Laboratory, ETH Zurich \\ Universitaetstr. 6, Zurich CH-8092, Switzerland \\ http://www.cse-lab.ethz.ch
}

\begin{abstract}
We present a novel approach for modeling subduction using a Multipole-accelerated Boundary Element Method (BEM). The present approach allows large-scale modeling with a reduced number of elements and scales linearly with the problem size. For the first time the BEM has been applied to a subduction model in a spherical planet with an upper-lower mantle discontinuity, in conjunction with a free-surface mesh algorithm.
\end{abstract}

Keywords: Boundary Elements, Subduction, Fluid Dynamics, Fast Multipole, Global Earth Model.

\section{Introduction}

Geodynamics at the planetary scale is an inherently multiscale problem exhibiting very large variations in viscosity and spatial scales. In a nearly isoviscous system, convection occurs through finger-like, cylindrically symmetric RayleighTaylor instabilities [1. In contrast, highly viscous plates do not sink, but drift laterally and sink asymmetrically at zones known as subduction zones. This subduction process is the driving mechanism of plate tectonics. A self-consistent model of plate tectonics involves flows at planetary scales (the pacific plate is over $10000 \mathrm{~km}$ wide) strongly coupled to small scale dynamics, such as trench fault lubrication (lithospheric shear-zones are thought to be $100 \mathrm{~m}$ to $1 \mathrm{~km}$ thick [2]).

Recent numerical results 34 have shown that the potential energy of the sinking plate is mostly dissipated through the interaction of the plate and the upper mantle. Accordingly a dynamic interaction between distinct slabs and the sharp viscosity transition at $660 \mathrm{~km}$ depth could be caused by mantle back flow. There is a debate as to whether, for example, these processes modify the kinematics, shape and stress state of the trenches of the ring of fire around the Pacific plate, or whether each subduction system can be considered independently.

The present work introduces a novel numerical approach based on a Fast Multipole (FM) extension of the Boundary Element Method(BEM). The resulting 
technique is much more efficient than the classical Finite Element (FE) or Finite Difference (FD) methods usually employed in geodynamics. We apply the present method to the simulation of subduction at the planetary scale.

This paper is structured as follows. In Sect. 2, we summarize our approach and present several resolution and scaling tests on a Beowulf cluster with up to 64 processors. In Sect. 3 then, we show how the Boundary Element Method for the solution of viscous flows can be adapted to subduction modeling. The results of models at the global scale are shown in Sect. 4

\section{Simulation of Stokes Flow}

\subsection{Governing Equations}

We consider the Stokes equation for a steady, highly viscous flow

$$
\nabla \cdot \sigma+\rho \mathbf{b}=0
$$

where $\sigma=-\nabla P+\mu \nabla^{2} \mathbf{u}$ is the stress tensor; $\mathbf{b}$, the body force; $P$, the pressure; $\mathbf{u}$, the velocity field and $\mu$, the dynamic viscosity.

The Stokes equation can be recast into a variety of integral equations. We follow here the formulation of [5], to which we refer for further details. We denote as $D$ is the domain where (1) holds and we write

$$
u_{i}\left(\mathbf{x}_{o}\right)=\frac{1}{8 \pi \mu} \int_{D} \sigma_{i k}(\mathbf{x}) n_{k} G_{i j}\left(\mathbf{x}, \mathbf{x}_{o}\right) d S(\mathbf{x})+\frac{1}{8 \pi} \int_{D} u_{i}(\mathbf{x}) n_{k} T_{i j k}\left(\mathbf{x}, \mathbf{x}_{o}\right) d S(\mathbf{x})
$$

where $G_{i j}$ and $T_{i j k}$ are the steady, Green's functions for velocity and stress respectively, also known as the Stokeslet and the Stresslet

$$
\begin{aligned}
G_{i j}\left(\mathbf{x}-\mathbf{x}_{o}\right) & =\frac{\delta_{i j}}{r}+\frac{\hat{x}_{i} \hat{x}_{j}}{r^{3}} ; \hat{\mathbf{x}}=\mathbf{x}-\mathbf{x}_{o} \text { and } r=|\hat{\mathbf{x}}| \\
T_{i j k}\left(\mathbf{x}-\mathbf{x}_{o}\right) & =-6 \frac{\hat{x}_{i} \hat{x}_{j} \hat{x}_{k}}{r^{5}} .
\end{aligned}
$$

In turn, (2) is cast into a form more appropriate for quasi-steady multiphase flows in the presence of a gravity field. Hence for $\mathrm{x} \in S_{i}$ we obtain

$$
\frac{1+\lambda_{i}}{2} \mathbf{u}(\mathbf{x})-\sum_{j}^{N} \frac{1-\lambda_{j}}{8 \pi} \int_{S_{j}}^{P V} \mathbf{n} \cdot \mathbf{T} \cdot \mathbf{u} d S=-\frac{1}{8 \pi \mu_{0}} \sum_{j}^{N} \int_{S_{j}} \mathbf{G} \cdot \Delta \mathbf{f} d S,
$$

where $P V$ denotes the principal value of the integral, $\mu_{0}$ is the viscosity of the mantle, taken as a reference and $\lambda_{i}=\mu_{i} / \mu_{0}$ and $\Delta \mathbf{f}$ is a normal stress jump that accounts for gravity

$$
\Delta \mathbf{f}=\Delta \rho \mathbf{b} \cdot \mathbf{x n} .
$$




\subsection{Numerical Method}

The surfaces $S_{i}$ and the supported quantities $\mathbf{u}, \Delta \mathbf{f}, \ldots$ are discretized with panels. The boundary integral equation (5) thus becomes a linear system

$$
((1+\lambda) / 2+\mathbf{T}) \mathbf{U}=\mathbf{F} .
$$

Many approaches rely to the construction of the matrix; this scales as $N_{\text {panels }}^{2}$ both memory- and computation time-wise though, making it impractical for large systems.

We use a fast multipole method (FMM) 6/7/8 for the evaluation of the integrals in (5). The FMM scales as $N \log (N)$, which is far more tractable and still allows the use of a Generalized Minimized Residual method (GMRES) or any Krylov space based method that does not rely on the storage of the full matrix.

A multipole method exploits the decay of the kernel to convolve and makes a controlled approximation. More explicitly, let us compute

$$
u\left(\mathbf{x}_{o}\right)=\int_{D} G\left(\mathbf{x}_{o}-\mathbf{x}\right) \rho(\mathbf{x}) d V(\mathbf{x}) .
$$

We consider the contribution from $D_{i}$, a part of $D$ that is far enough from our evaluation point $\mathbf{x}_{o}$ and proceed with a Taylor expansion of the kernel $G$ about $\mathbf{x}_{c} \in D_{i}$

$$
\begin{aligned}
u\left(\mathbf{x}_{o}\right)= & \int_{D_{i}} G\left(\mathbf{x}_{o}-\mathbf{x}\right) \rho(\mathbf{x}) d V(\mathbf{x}) \\
\simeq & \int_{D_{i}}\left(G\left(\mathbf{x}_{o}-\mathbf{x}_{c}\right)-\nabla G\left(\mathbf{x}_{o}-\mathbf{x}_{c}\right) \cdot\left(\mathbf{x}_{o}-\mathbf{x}_{c}\right)+\ldots\right) \rho(\mathbf{x}) d V(\mathbf{x}) \\
\simeq & G\left(\mathbf{x}_{o}-\mathbf{x}_{c}\right) \int_{D_{i}} \rho(\mathbf{x}) d V(\mathbf{x}) \\
& -\nabla G\left(\mathbf{x}_{o}-\mathbf{x}_{c}\right) \cdot \int_{D_{i}}\left(\mathbf{x}_{o}-\mathbf{x}_{c}\right) \rho(\mathbf{x}) d V(\mathbf{x})+\ldots
\end{aligned}
$$

We note that the equation involves successive moments of the $\rho$ distribution in $D_{i}$. The FMM algorithm thus sorts the sources in a tree structure whose cells contain the moment integrals-or multipoles- and carries out a field evaluation through a tree traversal. The refinement of the interactions is determined by a tree traversal stopping criterion based on a prescribed tolerance. The reader is referred to $[6] 7 / 8$ for further details.

The present FMM code can handle convolutions with the Green's functions for the Poisson equation, the Stokeslet or the Stresslet. It employs up to the second order moments of the source distributions (quadrupoles).

\subsection{Performances}

The FMM-BEM drastically improves the computational cost of the method. Figure 1(a) shows the computation time of a time step for a viscous sphere 


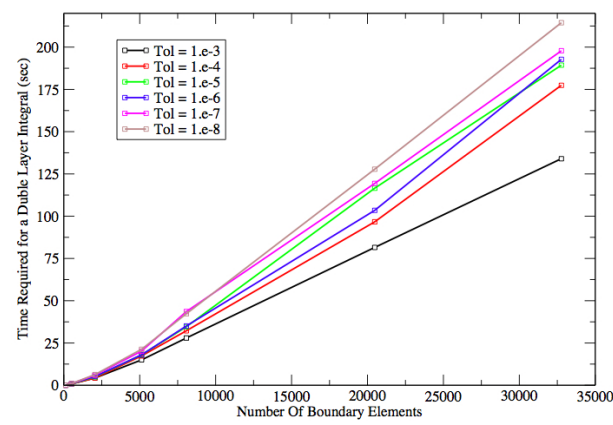

(a) Complexity

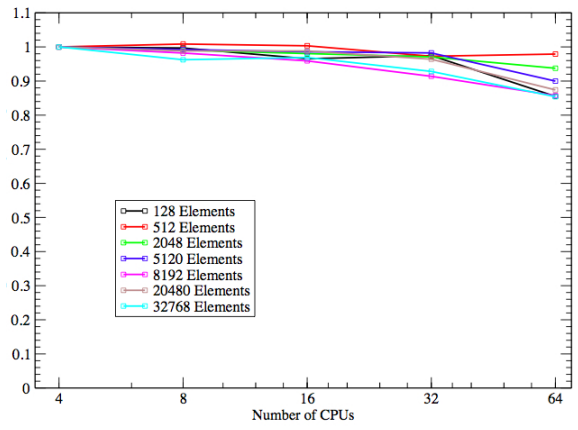

(b) Parallel efficiency

Fig. 1. Fast Multipole Method: performance plots

under gravity for several tolerances and resolutions. For the coarse resolutions, the method displays the nominal $N^{2}$ scaling of a direct interaction code. This is attributed to the relatively few elements and tree cells. The scaling then quickly approaches a nearly linear one $(N \log (N))$ for the finer resolutions.

The FMM-BEM has been parallelized using MPI. Figure 1(b) shows the parallel efficiency tested on a Opteron cluster with Quadrics connections. The scaling is very good up to 64 CPUS, still keeping $90 \%$ of efficiency. In its current implementation the FMM-BEM uses a shared tree, thus reducing the communication load at the expense of memory requirements.

\section{Modeling Subduction with FMM-BEM}

Subduction is the process through which a highly viscous $\left(O\left(10^{2}\right) \mu_{\mathrm{UM}}\right)$ and heavier plate $(1-3 \%)$ enters into the earth. Analytical and numerical models 910 have shown that the process is mainly controlled by the interaction of the lithosphere with the surface and is one-sided, characterized by an unsubducted plate overlapping the subducting plate. We assume that three fundamental forces control the subduction process: the lithosphere-mantle-air/water buoyancy difference, the viscous resistance of the mantle to the plate motion and the resistance to bending and stretching of the lithosphere itself.

Buoyancy difference arises from the density difference between the bottom and the top of the plate, of the mantle and air/water, respectively. This property is critical in subduction as it prevents the unsubducted plate from sinking straight down 11 .

The drag force exerted by the less viscous $\left(\mu_{\mathrm{UM}}\right)$ mantle on the lithosphere dissipates most of the energy 34 and controls the speed of the process. Consequently intraplate interaction might be mostly controlled by induced mantle flow.

The resistance to bending and stretching of the lithosphere itself is controlled by its very diverse and complex inner rheology. Simplified models of the internal distribution of strength of an oceanic lithosphere display a peak at $30-40 \mathrm{~km}$ 
depth, triggering the concept of lithospheric strength controlled by a stiff core [12/2/3. In this work, the overall resistance to bending and stretching will be parameterized by an effective uniform layer with a viscosity two orders of magnitude higher than the mantle and an effective thickness of $80 \mathrm{~km}$.

In order to appropriately model subduction, we have implemented a novel adaptive mesh algorithm which modifies the shape of the earth surface directly on top of the lithosphere. More explicitly, the algorithm adapts this free-surface to the lithospheric topography up to to a depth of half of the lithospheric thickness. The results of Sect. 4 show that this method automatically creates a physically consistent buoyancy force. The density difference between the mantle and ocean self-consistently sustains the unsubducted lithosphere as on the Earth but lets the upper surface of the lithosphere free to deform in a full free surface setting. Numerically, the method requires only one parameter, the critical distance $h_{\text {crit }}$ between the boundary elements of the Earth surface and of the lithosphere surface. If the distance is too low, instabilities arises during the computation and if it is too high the uplifting force is not properly calculated. Tests have shown that the critical distance $h_{c r i t}$ has to be no less than half of the element size of the Earth surface, but not more than $20 \%$ of the lithosphere thickness. The method is therefore more effective at a relatively high resolution, which is made affordable by the FMM-BEM.

\section{Results}

The simplest implementation of subduction at the global scale in a uniform Earth is obtained using only two surfaces, one delimiting the Earth surface and the second the lithosphere-mantle boundary. A slightly more sophisticated model, employing three surfaces, allows modeling the interaction with the $660 \mathrm{~km}$ discontinuity. We use the boundary integral form of (5), usually employed for solving the interaction between immersed distinct bodies, translating this setting to a bounded problem by orienting the normal of the Planet surface inwards, as sketched in Fig. 2 .

The reference viscosity $\mu_{0}$ of (5) is the upper mantle effective viscosity $\mu_{\mathrm{UM}}$; $\lambda_{1}=\mu_{\text {Ocean }} / \mu_{\mathrm{UM}}$ is virtually null, being the ratio between the viscosities of

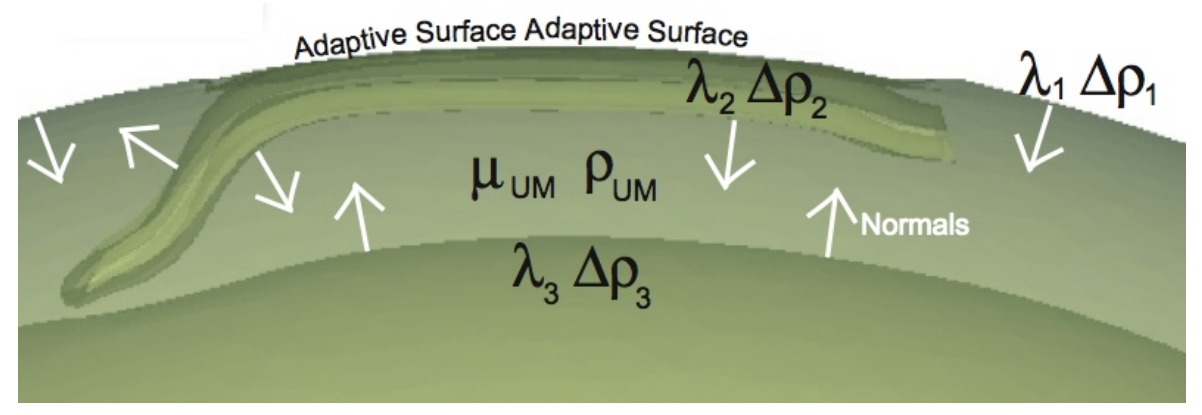

Fig. 2. Subduction model: geometry and parameters 


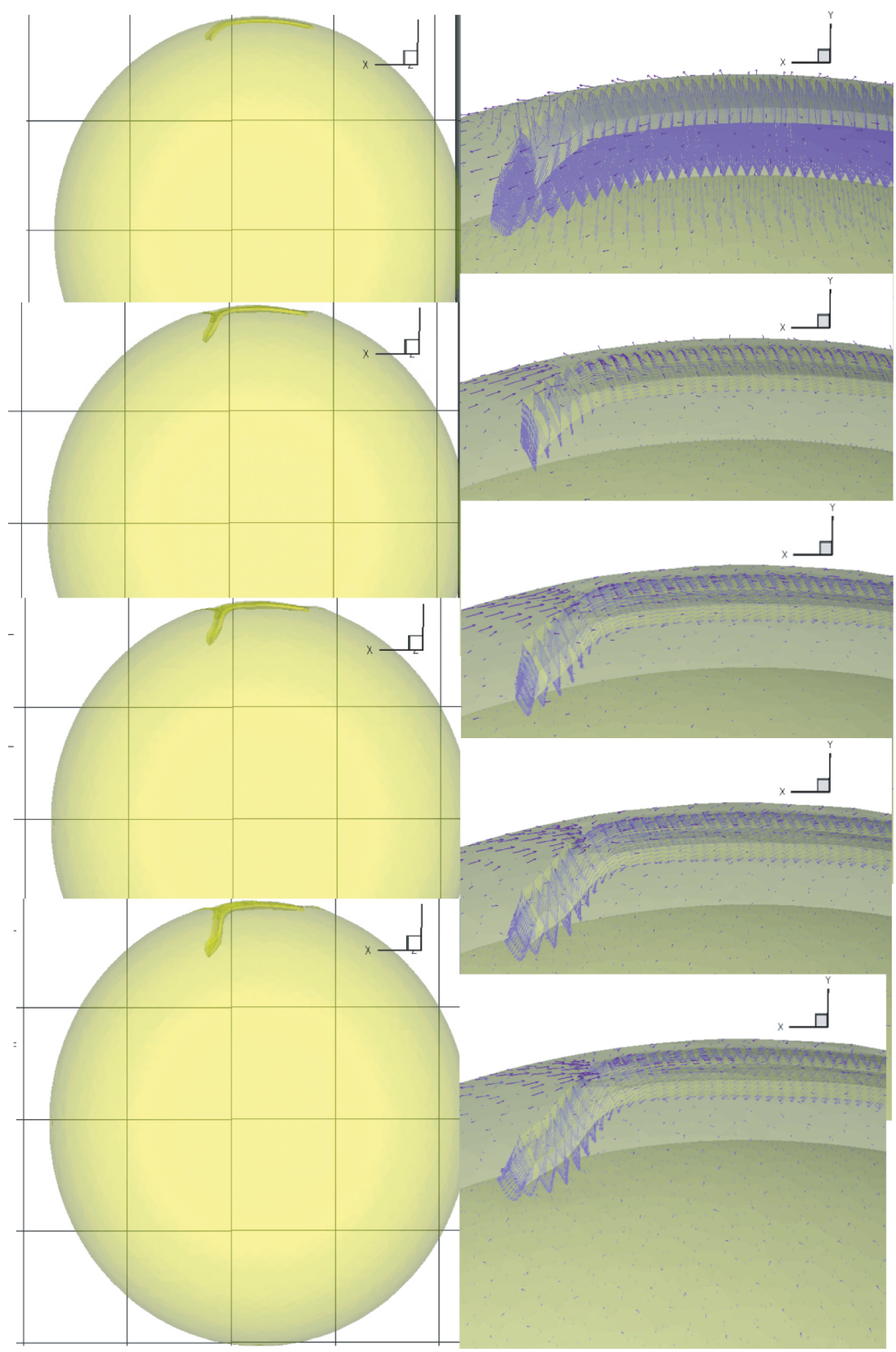

Fig. 3. Left: subduction of a plate 20 times more viscous then the mantle. Right: subduction of a plate 100 time more viscous then the mantle and interacting with a sharp viscous discontinuity at $660 \mathrm{~km}$, arrows represent the velocity vector. 
ocean (water) and the upper mantle; $\lambda_{2}=\mu_{\mathrm{Litho}} / \mu_{\mathrm{UM}}$ is the ratio between the lithosphere and upper mantle viscosities; $\lambda_{3}=\mu_{\mathrm{LM}} / \mu_{\mathrm{UM}}$ is the ratio between the lower and upper mantle viscosities. We define $\Delta \mathbf{f}$ on surfaces 1,2 and 3 in the same fashion; they are respectively equal to the difference between ocean (water) and upper mantle densities, the difference between the lithosphere and upper mantle densities and the difference between lower and upper mantle densities

$$
\begin{aligned}
\Delta \mathbf{f}_{1} & =\left(\rho_{\text {Ocean }}-\rho_{\mathrm{UM}}\right) g r \mathbf{n} \\
\Delta \mathbf{f}_{2} & =\left(\rho_{\text {Litho }}-\rho_{\mathrm{UM}}\right) g r \mathbf{n} \\
\Delta \mathbf{f}_{3} & =\left(\rho_{\mathrm{LM}}-\rho_{\mathrm{UM}}\right) g r \mathbf{n}
\end{aligned}
$$

where $r$ is the length of $\mathbf{x}, g$ is the gravity acceleration $\left(10 \mathrm{~m}^{2} / \mathrm{s}\right)$ and gravity is assumed constant and radial $\mathbf{b}=-g \mathbf{x} / r$.

The solution of equation [5] with the parameters described above is shown in Fig. 3. In the left column, four frames of the time evolution of subduction for a lithosphere $3 \%$ heavier and 20 times more viscous than the mantle are shown. Surface topography is amplified by a factor of 10 in order to better show the efficiency of the adaptive mesh algorithm to embed the buyancy force and selfconsistently let the planetary surface deform. Buoyancy sustains the lithosphere but allows the trench to freely migrate and the plate to freely move horizontally. At the beginning a 45 degrees dip is set, then the model evolves toward an almost vertical slab subduction, the dynamics accelerates, the trench retreat diminishes and the horizontal migration of the plate accelerates. Other models not shown here have been performed for two and more lithospheres just adding a new surface for each of them to equation 5 .

The right column of Fig. 3 shows four frames of the evolution of a lithosphere 100 times more viscous than the upper mantle subducting in an Earth with the $660 \mathrm{~km}$ (UM-LM) discontinuity. As for the first case, the plate is free to change shape and migrate horizontally and the trench evolves freely. Confirming results of laboratory experiments, the higher viscosity ratio and the presence of the upper-lower mantle discontinuity causes a lower subduction velocity but a much larger trench retreat than the first case. The arrows represent velocities, showing a slight forward motion for the plate while wedge velocities are much stronger. Finally, we note that the poloidal components of the velocities are dominant.

\section{Conclusions}

We have presented a novel computational approach for modeling subduction using a fast multipole acceleration of the boundary elements method. We have shown that the code scales linearly with the problem size for large sizes (more than $10^{3}$ elements) and shows a very good parallel behavior that is promising for larger systems. An adaptive mesh algorithm has been developed for reproducing the buoyancy force, within a free surface setting. Exploiting this innovation, for the first time FMM-BEM has been employed for modeling subduction at the planetary scale. Finally, illustrative examples have been presented for subduction 
in a viscously uniform Earth and in an Earth with the upper lower mantle viscosity discontinuity. Future work includes a comprehensive investigation of the roles played by the plate geometry, the lithosphere-mantle and upper-lower mantle differential properties and the study of the interaction of subducting plates.

\section{References}

1. Tackley, P.: Self-consistent generation of tectonic plates in time- dependent, threedimensional mantle convection simulations, 1. Pseudoplastic yielding. In: G3, Geochemistry Geophysics Geophysics (2000) Volume 1 Number 23

2. Regenauer-Lieb, K. and Yuen, D.A.: Modeling shear zones in geological and planetary sciences: solid- and fluid-thermal-mechanical approaches. In: Earth-Science Reviews (2003), Volume 63, 295-349

3. Capitanio, F.A., Morra G. and Goes, S.: Dynamic controls on subduction kinematics: The role of the downgoing lithosphere and passive mantle flow In: Earth and Planetary Science Letters, 2007 (now submitted)

4. Stegman, D.R., Freeman, J., Schellart, W. P., Moresi, L. and May, D.: Influence of trench width on subduction hinge retreat rates in 3-D models of slab rollback.

5. Pozrikidis, C.: Boundary Integral and Singularity Methods for Linearized Viscous Flow (1992).

6. Barnes, J. and Hut. P., A hierarchical $\mathrm{O}(\mathrm{N} \log \mathrm{N})$ force-calculation algorithm, Nature (1986), 324

7. Greengard, L. and Rokhlin, V.: A Fast Algorithm for Particle Simulations. In: Journal of Computational Physics A (1987), Volume 73, 325-348

8. Warren, M.S. and Salmon, J.K.: A parallel hashed Oct-Tree N-body algorithm. In: Supercomputing, 1993, Pages 12-21

9. Jacoby W.R.: Model Experiment of Plate Movements. In: Nature-Physical Science (1973), Volume 242, Number 122, 130-134

10. Christensen, U. R.: The influence of trench migration on slab penetration into the lower mantle. In: Earth and Planetary Science Letters (1996), Volume 1996, 27-39

11. Funiciello, F., Morra, G., Regenauer-Lieb, K. and Giardini, D.: Dynamics of retreating slabs (part 1): Insights from 2-D numerical experiments. In: Journal of Geophysical Research (2003), Volume 108(B4)

12. Regenauer-Lieb, K., Yuen, D. and Branlund, J.: The Initiation of Subduction: Criticality by Addition of water? In: Science (2001), Volume 294, 578-580 In: G3, Geochemistry Geophysics Geophysics (2006) Volume 7 Number 3 